https://doi.org/10.31426/ijamsr.2018.1.3.129

\title{
ANTERIOR ESTHETIC RESTORATIONS USING DIRECT COMPOSITE RESTORATION AND ALL CERAMIC VENEERS - 2 CASE REPORTS
}

\author{
Dr. Hunaid Arif Merchant ${ }^{1,}$ Dr. Swati Sharma ${ }^{2}$, Dr.Nikhita Devanathan ${ }^{3,}$ \\ Prof. Dr. Mithra N Hegde
}

Post Graduate Students, A.B. Shetty Memorial Institute Of Dental Sciences, Nitte (Deeemed To Be)
University, Mangaluru, Karnataka, India.
Professor and Head Of The Department, A.B. Shetty Memorial Institute Of Dental Sciences, Nitte

University, Mangalore India

mhunaid@gmail.com,

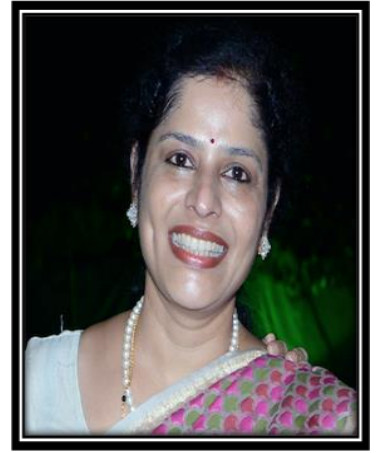

Prof.Dr. Mithra N. Hegde

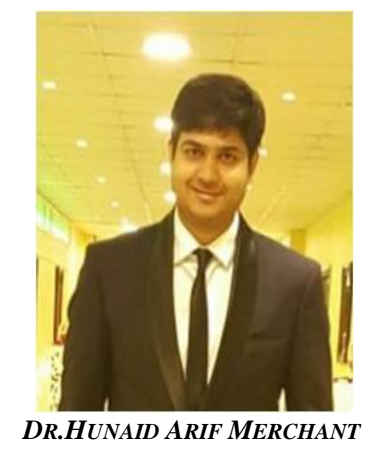

Keywords: All ceramic veneer, direct composite, anterior tooth fracture, discoloration

\begin{abstract}
A B S T R A C T
In esthetic dentistry, direct composite resins offer many advantages such as treatment in a single visit, not requiring preliminary models or wax-ups, not involving laboratory fees, cause less stress on the opposing dentition, and able to be repaired easily. Replacing tooth structure that is lost due to trauma to the anterior teeth remains a challenge to the restorative dentist. The restorations must not only be able to withstand masticatory forces, but also be esthetically pleasing and acceptable.Veneers describe the possibility of improving dental esthetics with low-thickness glass ceramics with conservation of tooth structure for patients with small to moderate anterior tooth material loss and little discoloration. This article presents two case reports where esthetics have been compromised and the use of direct composite restorations and all ceramic veneers as treatment options for restoring the aesthetic.
\end{abstract}

Citation: Dr. Hunaid Arif Merchant Dr. Swati Sharma Dr.Nikhita Devanathan' Prof. Dr. Mithra N Hegde (2018). Anterior Esthetic Restorations Using Direct Composite Restoration And All Ceramic Veneers - 2 Case Reports. International Journal of Advanced Multidisciplinary Scientific Research (IJAMSR) ISSN:2581-4281 Vol 1, Issue 3, May, 2018, \#Art.129, pp9-14

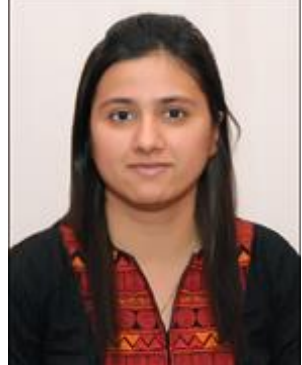

Dr.Swati Sharma

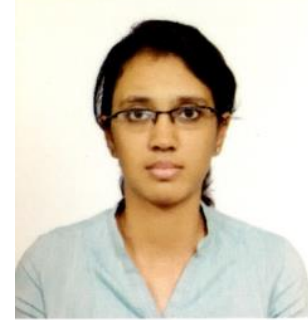

Dr. Nikhita Devanathan, 


\section{International Journal of Advanced Multidisciplinary Scientific Research (IJAMSR) ISSN:2581-4281}

\section{Introduction}

The esthetic treatment of anterior teeth has always presented a challenge in clinical practice. With the improvement of dental materials, many restorative options such as resin composites, all-ceramic crowns, and ceramic veneers have become available till date. In these circumstances, dentists and patients must choose the best alternative to improve oral conditions and esthetic results. ${ }^{1}$ Improved materials and techniques are being introduced in order to achieve better restorations while fulfilling patient esthetic expectations. ${ }^{2}$

The development of composite resins has advanced over the past decade, while the demand for esthetic direct restorations continues to grow. Moreover, they are easier to handle and insert, and facilitate the sculpture of the dental anatomy. 3 Facing these improvements, interventions with composite resins have made possible the re-establishment of specific individual details existing in the natural dentition, in a satisfactory esthetic way and practically imperceptible to layman vision. ${ }^{4}$

The idea of minimally invasive dental restorations is essential for successful restorations. ${ }^{5}$ Ceramic laminate veneers are indicated in the presence sufficient sound dental structure. This treatment option has been used due to the properties of ceramics like its color stability, biocompatibility, mechanical properties, and esthetics. ${ }^{6}$ Thus, minimum thickness ceramic laminate veneers have been increasingly indicated.

\section{Case 1:}

A male patient aged 24 years reported to the Department of Conservative Dentistry and Endodontics with a chief complaint of fractured upper front tooth since one year. On an intra oral examination, there was crowned fracture with respect to the left central incisor, involving enamel, dentin and in close approximation to the pulp (Figure 1). The tooth showed delayed response to electric pulp testing. Tenderness on percussion was negative, indicating the absence of any periapical inflammation. Radiographic examination revealed close approximation of the fracture line to the pulp with no root fracture (Figure 2). The treatment options available for the patient included pulp space therapy either followed by composite restoration or full coverage restoration and final closure of all the spaces by orthodontic treatment. Since there was no significant loss of tooth structure composite restoration was chosen as final restoration.

\section{Procedure for Pulp space therapy:}

Access cavity preparation was done under rubber dam isolation using Endo access bur followed by extirpation of pulp. After working length determination, biomechanical preparation was completed (Figure 3). The root canal was copiously irrigated using saline and sodium hypochlorite $(3 \%)$ alternatively. The canal was dried using paper points and obturation was done using the lateral condensation technique (Figure 4).

\section{Procedure for composite restoration:}

Shade selection was done prior to start of procedure (A2 Dentin and A2 enamel). The fractured incisal edge was bevelled using a tapered fissure diamond bur (Figure 5). Acid etching was carried out using $37 \%$ phosphoric acid for 20 seconds (Figure 6). Acid was rinsed away after 20 seconds and the tooth was dried using cotton. Bonding agent was applied in thin layers and light cured for 20 seconds. Incremental build- up of composite material was done (Figure 8). Finishing and polishing procedures were achieved using Soflex discs. (Figure 7).

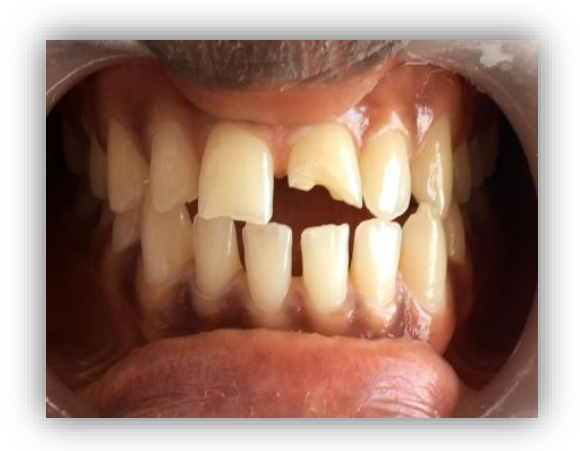

Figure 1: Intra oral examination revealing fracture involving enamel and dentin

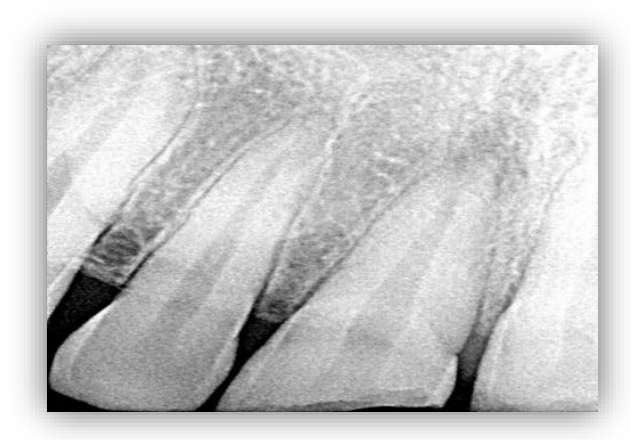

Figure 2: Pre-operative radiograph of the tooth 
https://doi.org/10.31426/ijamsr.2018.1.3.129

\section{International Journal of \\ Advanced Multidisciplinary Scientific Research (IJAMSR) ISSN:2581-4281} I J A M S R

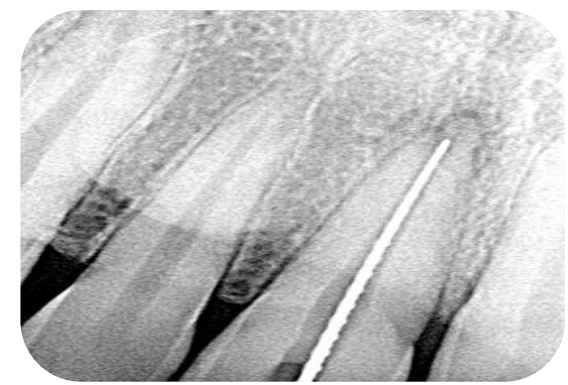

Figure 3: Working Length determination

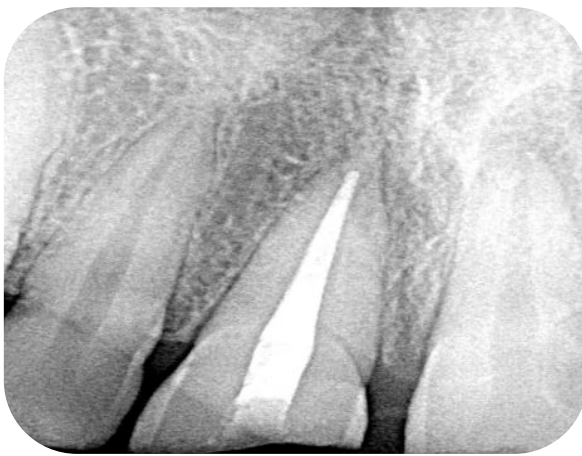

Figure 4: Post obturation radiograph

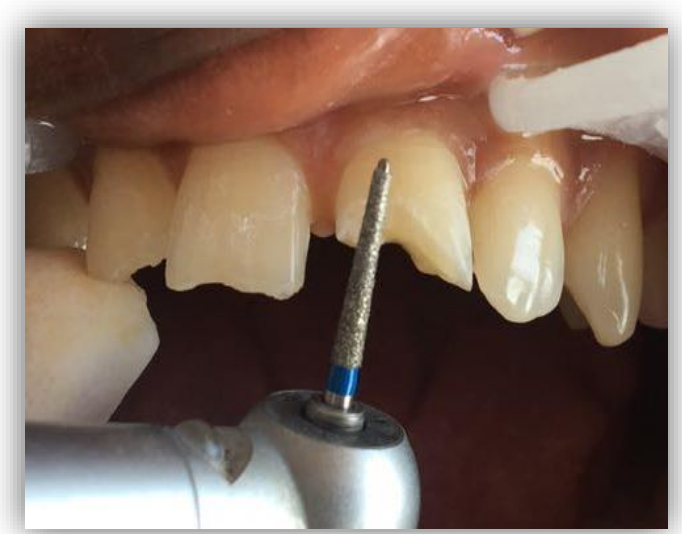

Figure 5: Preparation of the tooth structure

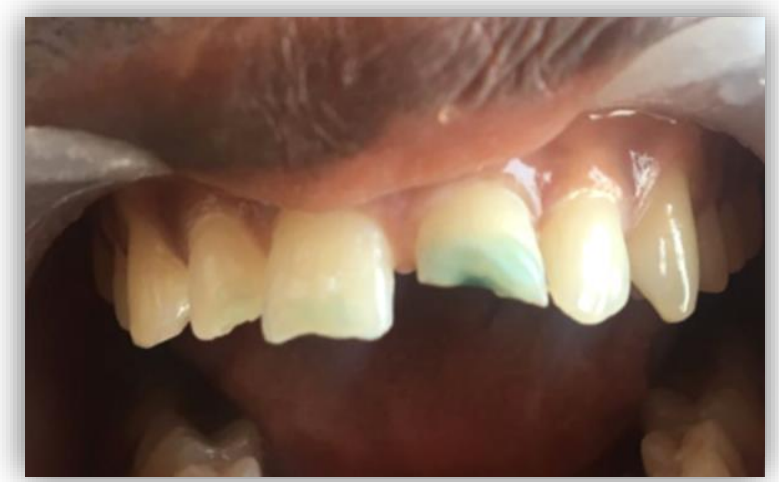

Figure 6: Acid etching of the prepared tooth surface

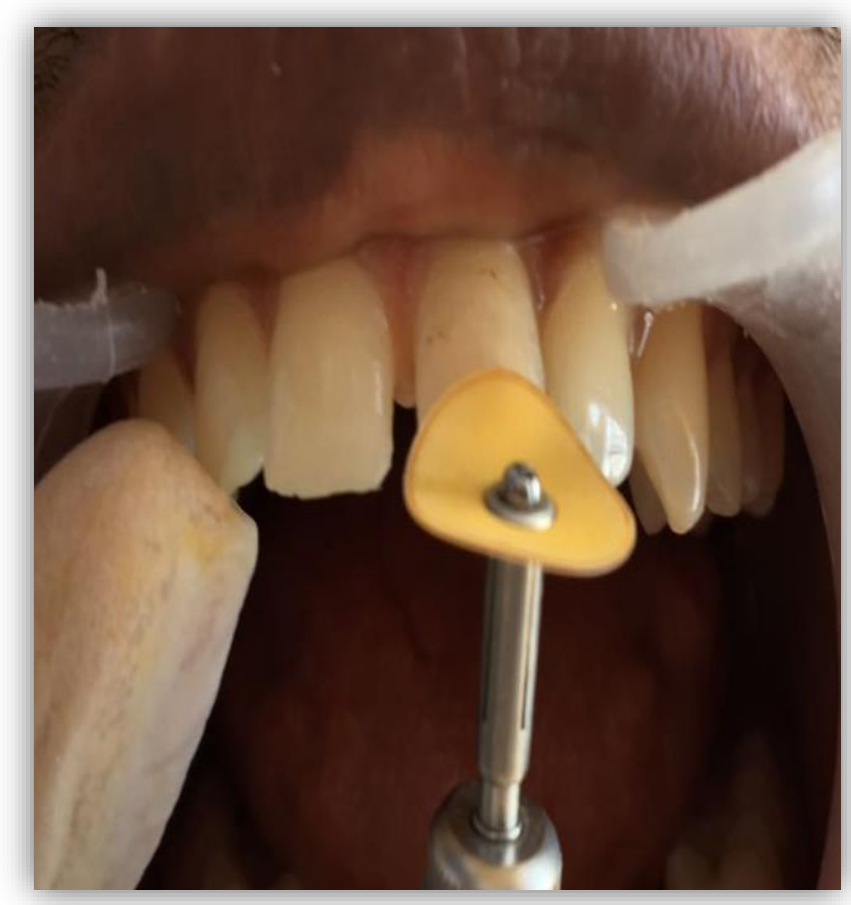

Figure 7: Finishing and polishing using Soflex discs 
https://doi.org/10.31426/ijamsr.2018.1.3.129

\section{J A M S R}

\section{International Journal of} Advanced Multidisciplinary Scientific Research (IJAMSR) ISSN:2581-4281
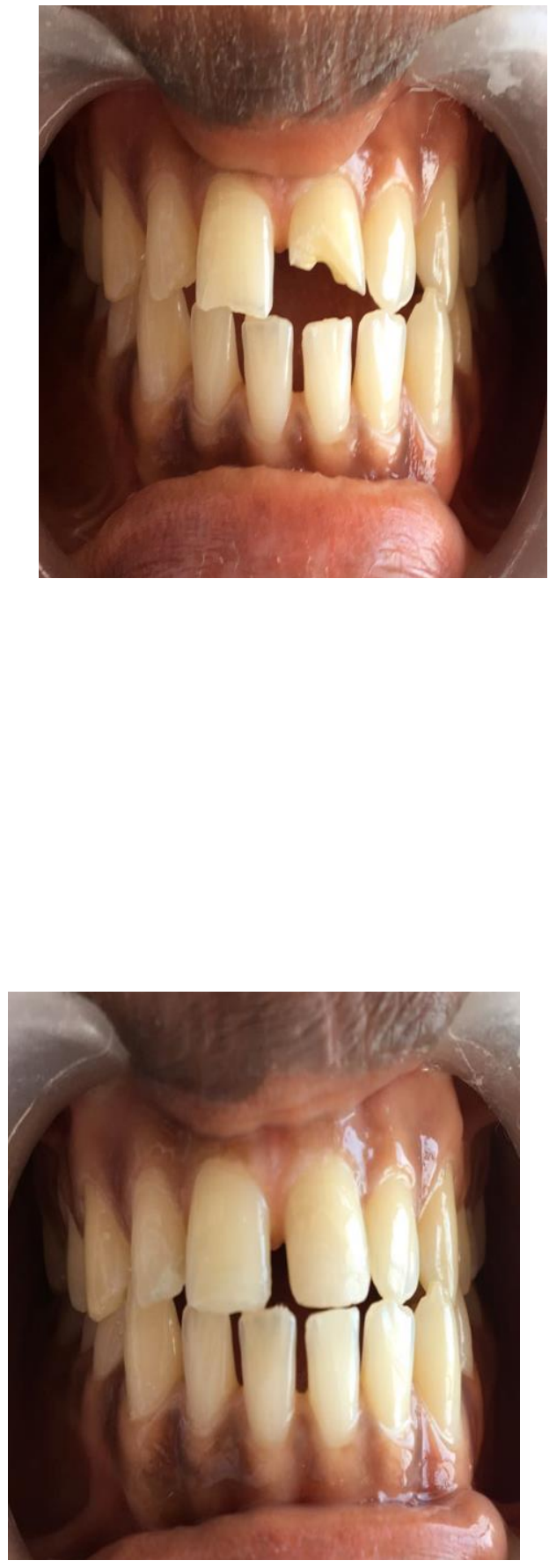

Figure 8: Comparison of Preoperative and Postoperative picture of the tooth 21
Case 2:

A 22 year old female patient reported to the department of Conservative Dentistry and Endodontics with the chief complaint of discolored upper front tooth in the mouth for 2 years. On an intra oral examination, the discoloration was seen with respect to tooth 11 . On radiographic examination calcification was seen in relation to 11. The treatment plan for this case was decided as the placement of all ceramic veneers.

Procedure: Before proceeding with the tooth preparation shade selection was done using a Vitaspan classical shade guide and A1 shade was chosen (Figure 9). The tooth preparation was carried out by keeping the depth of $0.5 \mathrm{~mm}$ in enamel at the incisal end and $0.3 \mathrm{~mm}$ at the cervical end (Figure 10). The incisal chamfer was extended palatally to produce the incisal lap design. The inner portion of veneer was etched carefully with $5 \%$ Hydrofluoric (HF) acid. After etching, it was washed thoroughly and dried with air spray. On drying, a coat of silane coupling agent was applied and air dried for 60 seconds. Bonding agent was applied onto the tooth surface and cured for 30 seconds (Figure 11). The veneer was then cemented using a resin cement (Figure 12).

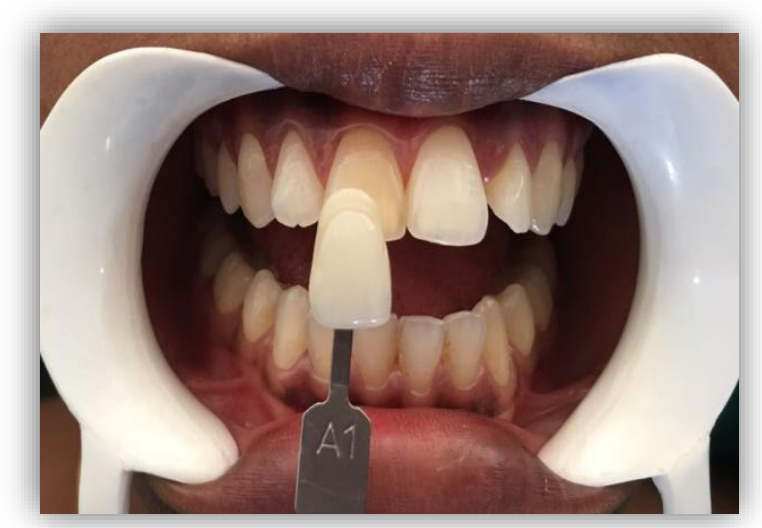

Figure 9: Shade selection using the Vitaspan Classic shade guide 
International Journal of Advanced Multidisciplinary Scientific Research(IJAMSR) ISSN:2581-4281 Volume 1, Issue 3, May,2018

\begin{tabular}{c|c}
\multicolumn{1}{c}{ https://doi.org/10.31426/ijamsr.2018.1.3.129 } \\
International Journal of \\
I J A M S R
\end{tabular}

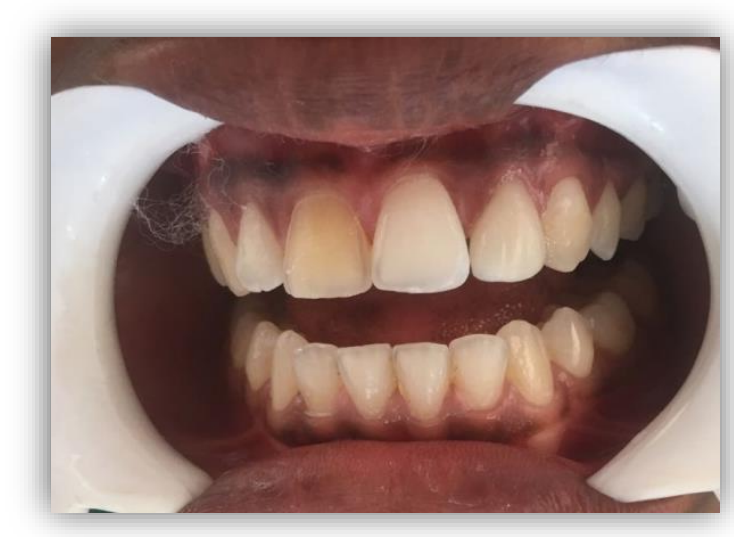

Figure 10: Tooth preparation for all ceramic veneers

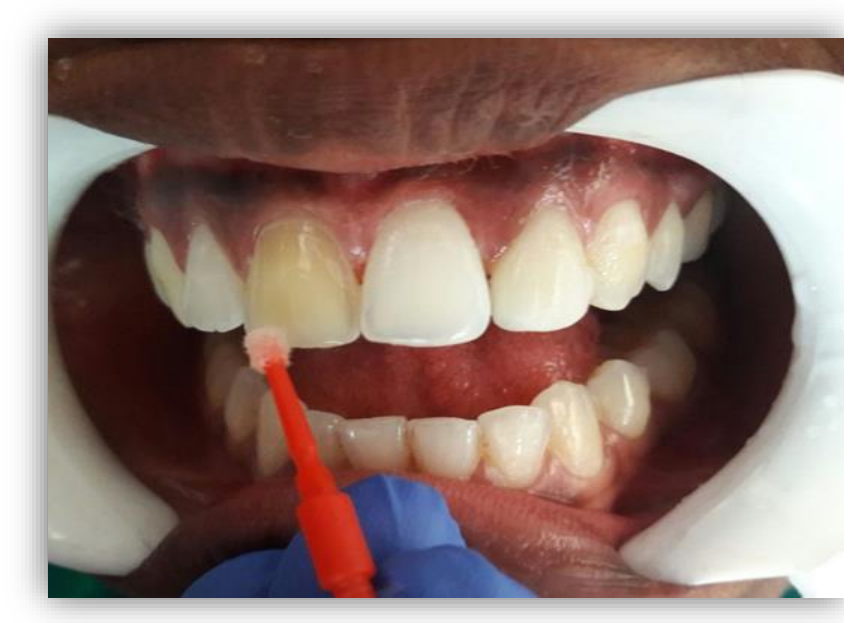

Figure 11: Application of bonding agent
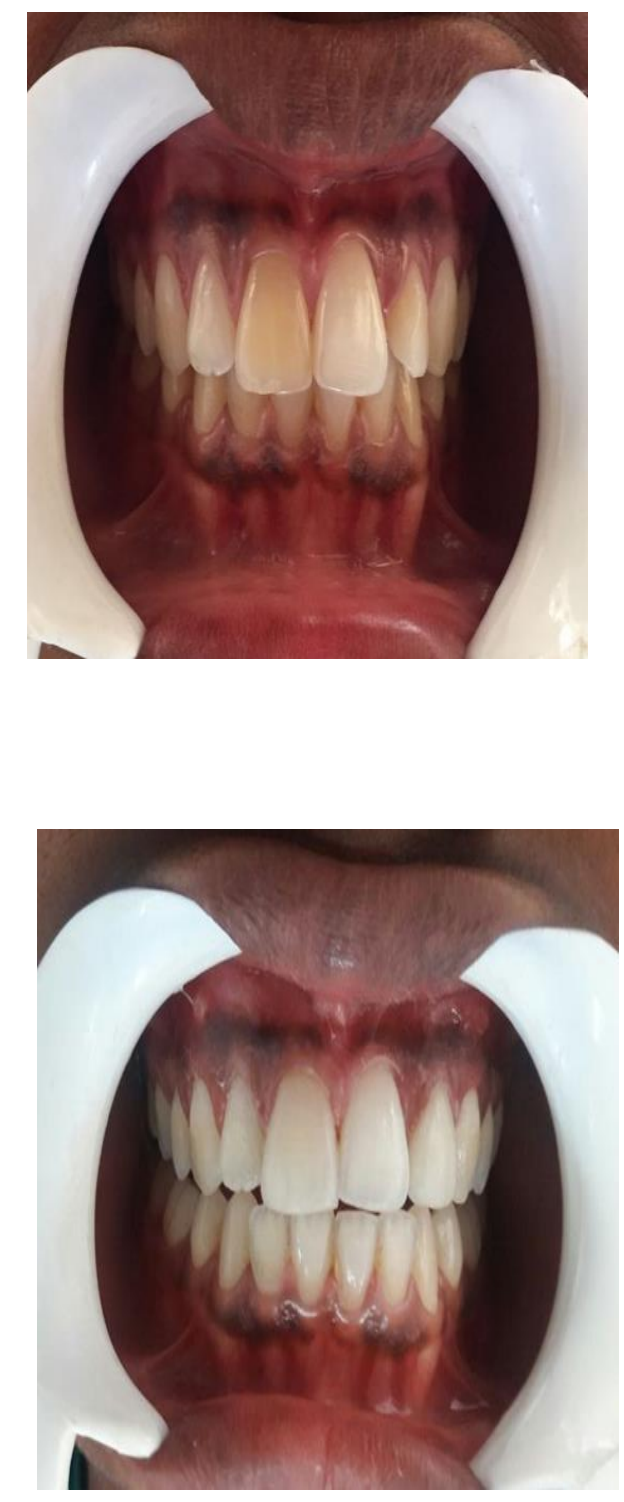

Figure 12: Preoperative and Postoperativeimages of the tooth 


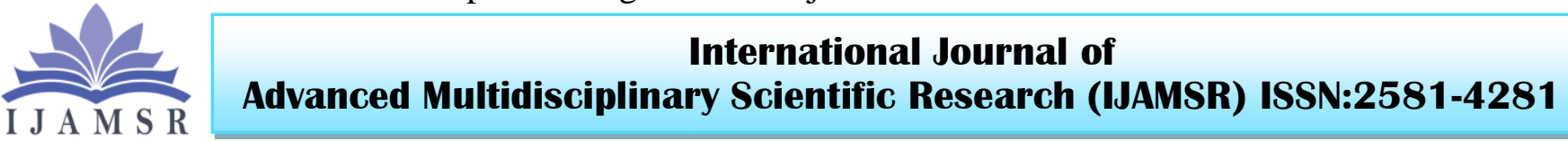

\section{DISCUSSION:}

Any restoration of the tooth must not only be able to withstand masticatory forces and stress, but also be esthetically pleasing and acceptable. Direct composite resins offer many advantages in esthetic dentistry Treatment can be completed in a single visit, do not require preliminary models or wax-ups, do not involve laboratory fees and cause less stress on the opposing dentition. ${ }^{7}$ Most of the composite materials possess less fractural resistance, less shear and compressive strength, and are not ideally suitable for high-stress areas. ${ }^{8}$ Parafunctional habits such as clenching and grinding, Class III malocclusion, large diastemas, or habits such as nail biting may possibly compromise the longevity of those restorations.

On the other hand, all ceramic veneers are used in cases where the there is sufficient amount of remaining tooth structure. These all ceramic veneers have their optical characteristic similar to the dental structure and good bonding. ${ }^{9}$ Moreover, when the dental preparation is restricted to the enamel, improved and more reliable bonding may be obtained.

In the first case, direct composite restoration was done. Since the fracture did not cause excessive loss of tooth structure, direct composite restoration was the preferred treatment option, also the final space closure will be achieved by orthodontic treatment. A three step layering technique with enamel shade in the outer labial and palatal surface and dentin shade for the inner surface was used. This was done to mimic the natural tooth, which represents the enamel translucency as well as the inner darker dentin.

In the second case, the restoration of the discolored tooth with all ceramic veneers was the preferred treatment option. The minimum thickness anterior ceramic, laminate veneers may be a conservative and better esthetic alternative to re-establish the form, shape, and color of anterior teeth. Since there was complete calcification of root canal, pulp space therapy was not carried out in this case and restoration was done using veneers.

\section{CONCLUSION:}

The ultimate success of esthetic treatment is only achieved when the patient is educated and motivated to maintain good oral health. The patient contribution and periodic follow up is imperative to the long-term success of the treatment.

\section{REFERENCES:}

1. da Cunha LF, Reis R, Santana L, Romanini JC, Carvalho RM, Furuse AY. Ceramic veneers with minimum preparation. European journal of dentistry. 2013 Oct; 7 (4): 492.

2. Qualtrough AJ, Burke FJ. A look at dental esthetics. Quintessence Int. 1994;25:7-14.

3. Direct adhesive restoration of anterior teeth: Part 2. Clinical protocol. de Araujo EM Jr, Baratieri LN, Monteiro S Jr, Vieira LC, de Andrada MA Pract Proced Aesthet Dent. 2003 Jun; 15 (5): 351-7; quiz 359.

4. Two-year follow-up of fractured anterior teeth restored with direct composite resin: report of three cases. Ozel E, Kazandag MK, Soyman M, Bayirli G Dent Traumatol. 2008 Oct; 24 (5): 589-92

5. Aesthetic changes with four anterior units. Bloom DR, Padayachy JN Br Dent J. 2006 Feb 11; 200 (3): 135-8.

6. All-ceramic systems: laboratory and clinical performance. Guess PC, Schultheis S, Bonfante EA, Coelho PG, Ferencz JL, Silva NR Dent Clin North Am. 2011 Apr; 55 (2): 333-52, ix.

7. Ionel DC, Luca R, Boazu D, Dobre M. Uncomplicated crown fractures-A finite element analysis of different treatment options. Annals of the University Dunarea de Jos of Galati: Fascicle II, Mathematics, Physics, Theoretical Mechanics. 2015; 38(1):137-141

8. Jordan RE. Esthetic composite bonding techniques and materials. St. Louis, Mosby-Year Book, Inc. 2nd ed. 1993;84-86, 132-134,140,150

9. Survival rates of all-ceramic systems differ by clinical indication and fabrication method. Land MF, Hopp CD J Evid Based Dent Pract. 2010 Mar; 10 (1): 37-8. 